\title{
Sensitivity Assessment of Wilms tumor gene (WT1) expression in Glioblastoma Using qPCR and Immunohistochemistry and its association with IDH1 mutation, and recurrence interval
}

Maher Kurdi ( $\sim$ Ahkurdi@kau.edu.sa )

King Abdulaziz University https://orcid.org/0000-0002-8979-3849

Nadeem Shafique Butt

King Abdulaziz University

\section{Saleh Baeesa}

King Abdulaziz University

Abudukadeer Kuerban

King Abdulaziz University

\section{Yazid Maghrabi}

King Faisal Specialist Hospital and Research Centre - Jeddah

\section{Anas Bardeesi}

King Faisal Specialist Hospital and Research Center

\section{Rothaina Saeedi}

King Abdulaziz University

\section{Badrah Alghamdi}

King Abdulaziz University

\section{Ahmed I. Lary}

King Abdulaziz Medical City - Jeddah

\section{Fawaz Mohammed}

King Abdulaziz University

\section{Research}

Keywords: Glioblastoma, IDH1 mutation, WT-1 Expression, Chemotherapies, PCR sensitivity

Posted Date: March 10th, 2021

DOl: https://doi.org/10.21203/rs.3.rs-290379/v1

License: (9) This work is licensed under a Creative Commons Attribution 4.0 International License. Read Full License 
Version of Record: A version of this preprint was published at Biologics: Targets and Therapy on July 16th, 2021. See the published version at https://doi.org/10.2147/BTT.S323358. 


\section{Abstract \\ Background}

The Wilms tumor 1 (WT1) gene has recently been shown to play a role in gliomagenesis, making it a potential immunotherapy target in glioblastomas. We aimed to investigate the most sensitive method to detect WT1 expression in glioblastoma and explore relationship between WT1 expression and isocitrate dehydrogenase-1 (IDH1) mutation.

\section{Methods}

Clinical and biological data were collected from 44 patients with totally resected glioblastomas, treated with adjuvant therapies, in the period between 2015 and 2019. WT1 protein expression was assessed in all cases using $\mathrm{IHC}$ while its gene expression was assessed in 13 clustered samples using quantitative polymerase chain reaction (qPCR). IDH1 mutation was assessed using immunohistochemistry (IHC). McNemar test was used to compare the sensitivity between IHC and RT-qPCR for WT1 gene expression detection. Kaplan Meier curves were used to compare the distribution of recurrence-free interval (RFI) between IDH1 and WT1 expression groups.

\section{Results}

The mean age was 54-years, with a male to female ratio 1.45. IDH1-wildtype was found in 26 cases (59.1\%) and the remining 18 cases $(40.9 \%)$ were IDH1-mutant. Through IHC, WT1 was overexpressed in 32 cases $(72.7 \%)$, partially expressed in 9 cases $(20.5 \%)$ and not expressed in only three cases $(6.8 \%)$. For the 13 cases tested by qPCR, 6 cases showed WT1 gene up-regulation and 7 cases showed WT1 downregulation. There was no significant difference in $W T 1$ expression among cases with different RNA concentrations regardless the testing method $(P$-value $<0.05)$. However, the difference between IHC and qPCR revealed $83 \%$ sensitivity, $28.5 \%$ specificity and $53 \%$ accuracy. IDH1-mutant cases with WT1 overexpression showed significant difference in recurrence interval $(P$-value $<0.048)$. This significance was not seen among IDH1-mutant cases with WT1 partial or no expression ( $P$-value $=0.56)$. There was also no significant difference in recurrence interval among IDH1-wildtype cases with WT1 partial or overexpression $(P$-value $=0.83)$.

\section{Conclusions}

Parallel testing for WT1 expression using IHC and qPCR is not reliable. However, IHC provides more accurate results than does $\mathrm{qPCR}$, which runs on fragmented tissue with indeterminant RNA concentrations. Moreover, IDH1-mutant glioblastomas with WT1 overexpression are associated with slow recurrence interval particularly if temozolomide with additional chemotherapies are used. 


\section{Introduction}

The Wilms tumor 1 (WT1) gene encodes a zinc finger transcriptional factor that plays an important role in cell growth and differentiation (1). WT1 been implicated in various malignancies. WT1 was first identified as a tumor suppressor because of frequent chromosome $11 \mathrm{p} 13$ region deletions observed in childhood renal neoplasm and Wilms tumor (2-3) and was then found to be overexpressed in leukemias and various solid tumors including breast and ovarian malignancies (4). Recent studies have shown that WT1 has a role in gliomagenesis (5). Consistently, WT1 overexpression has been found in high-grade gliomas (4) (6-7). However, the potential of using WT1 expression as a molecular marker has not been sufficiently substantiated. Recently, clinical trials of cancer immunotherapy targeting WT1 protein have shown promising results in glioblastomas, particularly in resistant cases. These results suggest that WT1 is a possible target for immunotherapy in high-grade gliomas (8) and also could increase the sensitivity of glioblastoma to chemoradiotherapy. The immunohistochemical approach is considered the standard method to detect WT1 protein expression. However, some studies have shown that WT1 mRNA levels are presenting similar results to that of the immunohistochemical score (4). Therefore, the most accurate method for testing WT1 expression in glioblastoma is not obviously clear. This study was designed to investigate whether IHC or qPCR are more for detecting $W T 1$ gene expression in glioblastoma cases.

Rauscher et al found that some high-grade gliomas lacked WT1 expression while Manocha et al identified an inverse relationship between WT1 scores and IDH1 mutation (9). They ascribed this negative expression in high-grade tumors to the younger age of patients and tumors possessing IDH1 mutations (10). Therefore, this study was also designed to explore the relationship between WT1 expression and IDH1 mutation and how this influences patient outcome.

\section{Material And Methods}

\section{Sample Stratification}

We included 44 patients with totally resected glioblastoma, who received adjuvant therapies, from one medical institution in Saudi Arabia between 2015 and 2019. Ethical approval for this study was granted by the National Biomedical Ethics Committee at King Abdulaziz University (HA-02-J-008) (Reference No. 189-19). Histological diagnoses were made according to the World Health Organization (WHO) classification. Clinical data including age at diagnosis, gender, post-operative adjuvant therapies, type of chemotherapies, and recurrence interval, were retrieved from hospital records. Patients were stratified based on IDH1 mutation and WT1 expression (Figure 1). Standard radiotherapy of a total dose of 60 Gy and temozolomide (TMZ) (150-200 mg/m² for 6-12 cycles) were administered to all patients at the time of management. Some patients received additional chemotherapies including etoposide, bevacizumab, irinotecan, and lomustine.

\section{Immunohistochemistry protocol used for IDH1 mutation assessment}


Anti-IDH1 antibody is intended for laboratory use to qualitatively identify IDH1 mutation in formalin fixed paraffin embedded tissue (FFPE) sections using an automated slide stainer. The IHC assay using antiIDH1 R132H (Clone H09) mouse monoclonal antibody was performed with an OptiView detection kit on a BenchMark XT automated staining system (Ventana). The assay procedure consisted of deparaffinization with EZ Prep at $75^{\circ} \mathrm{C}$, heat pre-treatment with Cell Conditioner for 68 minutes, and incubation with 1:20-1:50 diluted antibody for $32 \mathrm{~min}$ at $37^{\circ} \mathrm{C}$. Slides were counterstained with hematoxylin II and bluing reagent for 16 minutes. Sections in which > 10\% of tumor cells were positively stained were defined as mutant IDH1 (Figure 2).

\section{Assessment of WT1 expression through IHC and RT-qPCR}

\section{a. Assessment of WT1 protein expression using IHC}

Anti-Wilms tumor (WT1) antibody is intended for laboratory use to identify protein expression in FFPE sections on an automated slide stainer. The IHC assay using anti-WT1 (6F-H2) mouse monoclonal antibody was performed using the DAB detection kit from Ventana on a BenchMark XT automated staining system (Ventana). The assay procedure consisted of deparaffinization with EZ Prep at $75^{\circ} \mathrm{C}$, pretreatment with Cell Conditioner for 68 minutes, followed by incubation with 1:100-1:500 diluted antibody for $32 \mathrm{~min}$ at $37^{\circ} \mathrm{C}$. Slides were removed from the slide stainer after counterstaining with hematoxylin II and were immersed into successive alcohol buffer for 3 min. Sections in which tumor cell cytoplasm was positively stained were defined as "WT1 expressed". All 44 glioblastoma were stained for WT1 and the results were interpreted by a certified neuropathologist. The staining pattern was categorized as (i) overexpressed, (ii) partially expressed, and (iii) non-expressed (Figure 3).

\section{b. Assessment of WT1 gene expression using reverse transcriptase-qPCR}

Of the 44 enrolled glioblastoma cases, only 13 samples had adequate tissue and RNA quality for RT-PCR. This limitation should be taken into consideration during assessment of WT1 expression in clinical practice. The samples were divided into case $(n=13)$ and control $(n=2)$ groups. IHC results showed no WT1 expression in control cases. The reference gene used for RT-PCR was glyceraldehyde-3-phosphate dehydrogenase (GAPDH). Three replicates of threshold cycle $\left(\mathrm{C}_{\mathrm{T}}\right)$ values for five target genes and one reference gene were used for analysis. The mean $\mathrm{C}_{\mathrm{T}}$ and standard deviation for the reference $(G A P D H)$ and target (WT1) genes were calculated from the RT-PCR data and analyzed by $\Delta \Delta \mathrm{C}_{\mathrm{T}}$ and $\Delta \mathrm{C}_{\mathrm{T}}$ methods. The average $\mathrm{C}_{\mathrm{T}}$ for the control and each tested gene was calculated from the data generated by RT-PCR using the Step One System and Data Assist software. The outliers were omitted from analysis. The $\mathrm{C}_{\mathrm{T}}$ of the target gene was normalized to the $\mathrm{C}_{\mathrm{T}}$ of the reference gene, then the $\Delta \mathrm{C}_{\mathrm{T}}$ of the test sample was normalized to the $\Delta \mathrm{C}_{\mathrm{T}}$ of the control sample and the relative quantification $(\mathrm{Rq})$ and differential expression (fold change, FC) were calculated using:

(1) $\Delta \mathrm{C}_{\mathrm{T}}$ for Ctrl or test $=\mathrm{C}_{\mathrm{T}}$ target gene $-\mathrm{C}_{\mathrm{T}}$ reference gene (2) $\Delta \Delta \mathrm{C}_{\mathrm{T}}=\Delta \mathrm{C}_{\mathrm{T}}$ test sample $-\Delta \mathrm{C}_{\mathrm{T}}$ control sample, (3) Relative quantification $(\mathrm{Rq})=2^{-\Delta \Delta C T}=$ value*, however, $\mathrm{Rq}>1$ represents up-regulation 
whereas $\mathrm{Rq}<1$ represents down regulation of gene.

The fold change (differential expression) was also calculated. $\Delta \mathrm{C}_{\mathrm{T}}$ values for each sample were determined using: $\Delta \mathrm{C}_{\mathrm{T}}=\left[\right.$ mean $\mathrm{C}_{\mathrm{T}}$ reference gene - mean $\mathrm{C}_{\mathrm{T}}$ target gene (Table 1).

\section{Statistical Methods}

Data are described as frequencies and percentages. The McNemar test was used to compare the sensitivity, specificity, and accuracy of IHC and RT-qPCR for WT1 gene expression detection. Kaplan Meier curves were used to compare the distribution of recurrence-free interval (RFI) between mutant IDH1 and WT1 expression groups. Recurrence interval (RI) is defined as the period after total surgical resection to the first possible date of recurrence. All statistical analyses were performed using IBM SPSS1 ver. 24 statistical software programs (SPSS Inc., Chicago, IL).

\section{Results}

Forty-four patients with resected and treated glioblastoma were included in this study. The mean patient age was 54-years, with a male to female ratio 1.45. Parietal and frontal areas were the predominant tumor location and tumors in these locations were observed in 33 cases (75\%). Wildtype IDH1 was found in 26 cases $(59.1 \%)$ and the remining 18 cases $(40.9 \%)$ contained mutant IDH1. IHC revealed WT1 overexpression in 32 cases (72.7\%), partial expression in nine cases $(20.5 \%)$, and no expression in three cases (6.8\%). For the 13 cases in which WT1 expression was tested using QPCR, six and seven cases had up- and down-regulated WT1 expression, respectively (Table 1-2). Forty one patients (93.2\%) received chemoradiotherapy and three patients did not receive any adjuvant therapy. Among patients who received chemotherapies, around 52\% $(n=23)$ were treated with TMZ alone and $27 \%(n=17)$ were treated with TMZ and additional chemotherapeutic agents. The mean recurrence time was 579 days after the total surgical resection of the tumor. Approximately $36 \%(n=16)$ of patients had tumor recurrence within or after 1-year of resection while $63.6 \%(n=28)$ showed recurrence before 1-year of resection. Table 2 summarizes the descriptive distribution of the data.

\section{IHC and RT-qPCR detection of WT1 expression in patients with glioblastoma}

There was no clear evidence in the literature about whether IHC or qPCR provide better results when measuring WT1 expression. However, RT-qPCR can still be run using severely fragmented tissue but the RNA content is often barely enough to provide beneficial results.

We investigated the accuracy of both testing methods assuming that IHC, based on the previous published data, is more accurate. First, with the exception of 13 samples, most FFPE tissue from cases included in this analysis had a low RNA concentration were excluded from PCR. The remaining 13 samples had RNA concentrations $>20 \mathrm{~nm}$. IHC and qPCR were used to examine and compare WT1 expression in these 13 samples. Two cases showed downregulation (no expression) in both $\mathrm{IHC}$ and qPCR. A single case, with low RNA concentration (22.7 nm) showed no WT1 expression via IHC and WT1 
up-regulation by qPCR. Five cases that showed WT1 overexpression on IHC had qPCR results showing downregulation. The remaining five cases had parallel results. There was no significant difference in WT1 expression status between samples with different RNA concentrations $(P$-value $<0.05)$. The McNemar test revealed that $83 \%$ sensitivity and $28.5 \%$ specificity were achieved using IHC rather than $\mathrm{QPCR}$ for assessing WT1 expression (Table 3). The lack of significant difference in WT1 expression results between samples tested with IHC and qPCR indicates that both methods are not reliable to be in parallel.

\section{Relationship between IDH1 status, WT1 expression, and recurrence interval}

The recurrence interval among patients with $W T 1$ overexpression significantly differs between those with wildtype and mutant IDH1 ( -value < 0.048). Cases with mutant IDH1 showed late recurrence, after 1-year (Figure 4). This significance was not observed among cases with mutant IDH1 and partially expressed or overexpressed WT1 ( $P$-value $=0.56)$. Together, these results indicate that, in cases with mutant IDH1, WT1 up-regulation lowers the chance of tumor recurrence. No significant difference in recurrence interval was observed among cases with wildtype IDH1 and WT1 partial or overexpression ( $P$-value $=0.83$ ) (Figure 4). Moreover, patients with glioblastoma with IDH1 mutation and WT1 overexpression who had received TMZ and additional chemotherapies showed better survival rates and longer recurrence intervals than did those who received TMZ alone. However, no significant difference in tumor recurrence interval was observed among patients with mutant IDH1 and WT1 overexpression who received either TMZ alone or TMZ and additional therapies $(P$-value $=0.19)$.

\section{Discussion}

Glioblastoma is the most aggressive primary malignant brain tumor in adults. While primary and secondary glioblastomas are pathologically indistinguishable, they vary at the molecular level. After surgical resection, current standard treatment for patients with glioblastoma is combined radiotherapy and chemotherapy using either TMZ alone or TMZ with additional chemotherapeutic agents. The overall survival rate (OS) for patients with glioblastoma is around 14.6 months with a 5-year long-term survival. Nevertheless, glioblastoma remains a fatal disease and treatment strategies are palliative, and aim to improve survival rate and maintain a good quality of life.

WT1 encodes a zinc finger transcriptional factor and plays an important role in cellular growth and differentiation (1). WT1 has been implicated in various malignancies and was first identified as a tumor suppressor gene due to frequent deletions of chromosome region $11 \mathrm{p} 13$ in childhood renal neoplasm (WT) (2-3). WT1 was found to be overexpressed in leukemias and solid tumors including breast and ovarian malignancies (4). Recent studies have shown that WT1 plays a role in gliomagenesis (5). WT1 overexpression has been repetitively observed in high-grade gliomas (4) (6-7). However, the utility potential of WT1 expression as a biomarker has not been sufficiently substantiated. Testing WT1 gene or protein expression in patients with glioblastoma patients is important for planning treatment and prognostic determination. Recently, clinical trials of cancer immunotherapies targeting WT1 have shown promising results in glioblastomas, particularly in resistant cases, suggesting that $W T 1$ is a potential 
target for immunotherapy in high-grade gliomas (8) and increases the sensitivity of glioblastoma to chemoradiotherapy.

Although the immunohistochemical approach for assessing WT1 expression is a useful method, some studies have shown similar WT1 expression results using both ICH and molecular analyses (4). Our results showed that there is no difference in $W T 1$ expression results using different tests with different RNA concentrations. However, low RNA volume and concentration may give false qPCR results. This does not happen when WT1 expression being tested by IHC. Our analysis revealed that IHC has $83 \%$ sensitivity, $28.5 \%$ specificity, and $53 \%$ accuracy supporting the notion that IHC is a reliable test for WT1 expression. Moreover, our results show that parallel testing of WT1 expression using IHC and QPCR is not reliable and that IHC provides more accurate results than does QPCR.

The association between IDH1 mutation and WT1 expression has also not been thoroughly investigated to date. Manocha et al identified an inverse relationship between WT1 scores and IDH1 mutation (9). Rauscher et al found that some anaplastic astrocytomas and glioblastoma lack WT1 expression. They ascribed this lack of WT1 expression in high-grade tumors to the younger age of patients and the presence of IDH1 mutations in the tumors (10). In our analysis, we found that glioblastomas with mutant IDH1 and WT1 overexpression are associated with longer tumor recurrence interval compared with cases with wildtype IDH1. The association between WT1 expression and the type of chemotherapy administered has also never been studied to date. Although WT1 immunotherapy is currently under trial, we found that TMZ taken with additional chemotherapies may improve the survival rate and decrease the chance of tumor recurrence in patients with glioblastomas. This means that TMZ with other chemotherapies may be used as an add-on to WT1 immunotherapy to prevent tumor regression, increase sensitivity to TMZ, and decrease tumor recurrence. Further research should be conducted in this area.

\section{Declarations}

Ethical approval: Ethical approval for this study was granted by the National Biomedical Ethics Committee at King Abdulaziz University (HA-02-J-008) (Reference No. 189-19).

Consent for publication: ready on request

Availability of the data: The datasets used and/or analysed during the current study are available from the corresponding author on reasonable request.

Competing of interest: The authors declare that they have no competing interests" in this section.

Funding: The study is sponsored and funded by "Deanship of Scientific Research of King Abdulaziz University, Jeddah, Saudi Arabia” (Code No. G: 81-828-1441).

\section{Authors contribution:}

MK, idea, IRB submission, writing, study design and data, histological analysis. 
NS, Statistical analysis

SB, data provider, writing, analysis

AK, study design, statistical analysis, PCR analysis, writing, editing,

YM, data entry, tissue collection, writing

$A B$, data entry, tissue collection

RS, data entry, tissue collection, writing

BG, data analysis, editing, submission, consultation

$\mathrm{AL}$, data provider, IRB submission

FM, Tissue collection, IRB submission, IHC

\section{Acknowledgment: $n / a$}

\section{References}

1. Pritchard-Jones K. The Wilms tumor gene, WT1, in normal and abnormal nephrogenesis. Pediatr Nephrol 1999; 13: 620-625.

2. Call KM, Glaser T, Ito CY, Buckler AJ, Pelletier J, Haber DA, et al. Isolation and characterization of a zinc finger polypeptide gene at the human chromosome 11 Wilms' tumor locus. Cell 1990; 60: 509520.

3. Nakatsuka S, Oji Y, Horiuchi T, Kanda T, Kitagawa M, Takeuchi T, et al. Immunohistochemical detection of WT1 protein in a variety of cancer cells. Mod Pathol 2006; 19: 804-814.

4. Schittenhelm J, Beschorner R, Simon P, Tabatabai G, Herrmann C, Schlaszus H, et al. Diagnostic value of WT1 in neuroepithelial tumor. Neuropathol Appl Neurobiol 2009; 35: 69-81.

5. Clark AJ, Ware JL, Chen MY, Graf MR, Van Meter TE, Dos Santos WG, et al. Effect of WT1 gene silencing on the tumorigenicity of human glioblastoma multiforme cells. J Neurosurg 2010; 112: 1825.

6. Kijima N, Hosen N, Kagawa N, Hashimoto N, Kinoshita M, Oji Y, et al. Wilms' tumor 1 is involved in tumorigenicity of glioblastoma by regulating cell proliferation and apoptosis. Anticancer Res 2014; 34: $61-67$.

7. Somasundaram A, Ardanowski N, Opalak CF, Fillmore HL, Chidambaram A, Broaddus WC. Wilms tumor 1 gene, CD97, and the emerging biogenetic profile of glioblastoma. Neurosurg Focus 2014; 37: 1-4.

8. Izumoto S, Tsuboi A, Oka Y, Suzuki T, Hashiba T, Kagawa N, et al. Phase II clinical trial of Wilms tumor 1 peptide vaccination for patients with recurrent glioblastoma multiforme. J Neurosurg 2008; 
108: $963-971$.

9. Manocha a, Jain S. WT1 in astrocytomas: comprehensive evaluation of immunohistochemical expression and its potential utility in different histological grades. Indian J Cancer 2019; 56: 197201.

10. Rauscher J, Beschorner R, Gierke M, Sotirios B, et al. WT1 expression increases with malignancy and indicates unfavourable outcome in astrocytoma. J Clin Pathol. 2014; 67: 556-561.

\section{Tables}

Table 1. WT1 gene expression as measured using IHC and RT-qPCR.

\begin{tabular}{|lllllll|}
\hline & $\begin{array}{l}\text { WT1 Expression } \\
\text { (IHC) }\end{array}$ & $\begin{array}{l}\text { WT1 Expression } \\
\text { (qPCR) }\end{array}$ & $\begin{array}{l}\text { WT1 C } \\
\text { mean }\end{array}$ & $\begin{array}{l}\text { GDPH C } \\
\text { mean }\end{array}$ & $\begin{array}{l}\text { FCCT } \\
\text { WT1 }\end{array}$ & Rq \\
\hline 1 & Overexpressed & Upregulated & 39.47 & 32.14 & 3.42 & 3.42 \\
\hline 2 & Overexpressed & Upregulated & 36 & 29.19 & 4.9 & 4.9 \\
\hline 3 & $\begin{array}{l}\text { Partially } \\
\text { expressed }\end{array}$ & Upregulated & 35.01 & 31.7 & 55.62 & 55.62 \\
\hline 4 & Not expressed & Upregulated & 35.64 & 28.89 & 5.42 & 5.42 \\
\hline 5 & Overexpressed & Upregulated & 36.53 & 27.65 & 1.17 & 1.17 \\
\hline 6 & Overexpressed & Upregulated & 34.98 & 31.21 & 40.55 & 40.55 \\
\hline 7 & Overexpressed & Down regulated & 37.5 & 19.9 & -361 & 0.0028 \\
\hline 8 & Not expressed & Down regulated & 36.42 & 22.97 & -20.25 & 0.05 \\
\hline 9 & Overexpressed & Down regulated & 37.5 & 23.8 & -24.49 & 0.04 \\
\hline 10 & Overexpressed & Down regulated & 36.7 & 19.5 & -280 & 2.76 \\
\hline 11 & Not expressed & Down regulated & 37.47 & 28.08 & -1.22 & 0.82 \\
\hline 12 & Overexpressed & Down regulated & 38.95 & 21.3 & -374 & 0.0027 \\
\hline 13 & Overexpressed & Down regulated & 39.98 & 23.25 & -197 & 0.0051 \\
\hline
\end{tabular}

CT: threshold cycle $\left(C_{T}\right)$, GAPDH: glyceraldehyde-3-phosphate dehydrogenase; Rq: Relative quantification.

Table 2. Patients data. 


\begin{tabular}{|c|c|}
\hline & Overall $(n=44)$ \\
\hline \multicolumn{2}{|l|}{ Age } \\
\hline Mean (SD) & $54.8(15.3)$ \\
\hline Range & $11.0-82.0$ \\
\hline \multicolumn{2}{|l|}{ Gender } \\
\hline Female & $18(40.9 \%)$ \\
\hline Male & $26(59.1 \%)$ \\
\hline \multicolumn{2}{|l|}{ Tumour Location } \\
\hline Frontal & $16(36.4 \%)$ \\
\hline Occipital & $3(6.8 \%)$ \\
\hline Parietal & $17(38.6 \%)$ \\
\hline Temporal & $8(18.2 \%)$ \\
\hline \multicolumn{2}{|l|}{ IDH1 Status } \\
\hline IDH-mutant & $18(40.9 \%)$ \\
\hline IDH-wildtype & $26(59.1 \%)$ \\
\hline \multicolumn{2}{|l|}{ WT1 Expression (IHC) } \\
\hline Not expressed & $3(6.8 \%)$ \\
\hline Overexpressed & $32(72.7 \%)$ \\
\hline Partially expressed & $9(20.5 \%)$ \\
\hline \multicolumn{2}{|l|}{ Adjuvant Therapy } \\
\hline Chemoradiotherapy & $41(93.2 \%)$ \\
\hline None & $3(6.8 \%)$ \\
\hline \multicolumn{2}{|l|}{ Chemotherapy Type } \\
\hline Temozolomide & $23(52.3 \%)$ \\
\hline Temozolomide Other & $18(41 \%)$ \\
\hline \multicolumn{2}{|l|}{ Recurrence Interval } \\
\hline Mean (SD) & $579.3(348.8)$ \\
\hline Range & $61.0-1344.0$ \\
\hline Recurrence Time & \\
\hline
\end{tabular}




\begin{tabular}{|ll|}
\hline & Overall $(\mathbf{n = 4 4 )}$ \\
\hline < one Year & $28(63.6 \%)$ \\
\hline > one Year & $16(36.4 \%)$ \\
\hline
\end{tabular}

Table 3. McNemar test was used to detect matching compatibility between expression results obtained using $\mathrm{IHC}$ and $\mathrm{qPCR}$.

\begin{tabular}{|c|c|c|c|c|}
\hline \multicolumn{5}{|c|}{ WT1 expression measured by qPCR } \\
\hline WT1 Expression (IHC) & Down regulated & Upregulated & Total & $P$-value \\
\hline Down regulated & 2 & 1 & 3 & $0.102^{\times 2}$ \\
\hline Upregulated & 5 & 5 & 10 & $0.221^{\times 2 c}$ \\
\hline Total & 7 & 6 & 13 & \\
\hline
\end{tabular}

\begin{tabular}{|ll|}
\hline Sensitivity & $83.3 \%$ \\
\hline Specificity & $28.6 \%$ \\
\hline Accuracy & $53.8 \%$ \\
\hline
\end{tabular}

Figures 


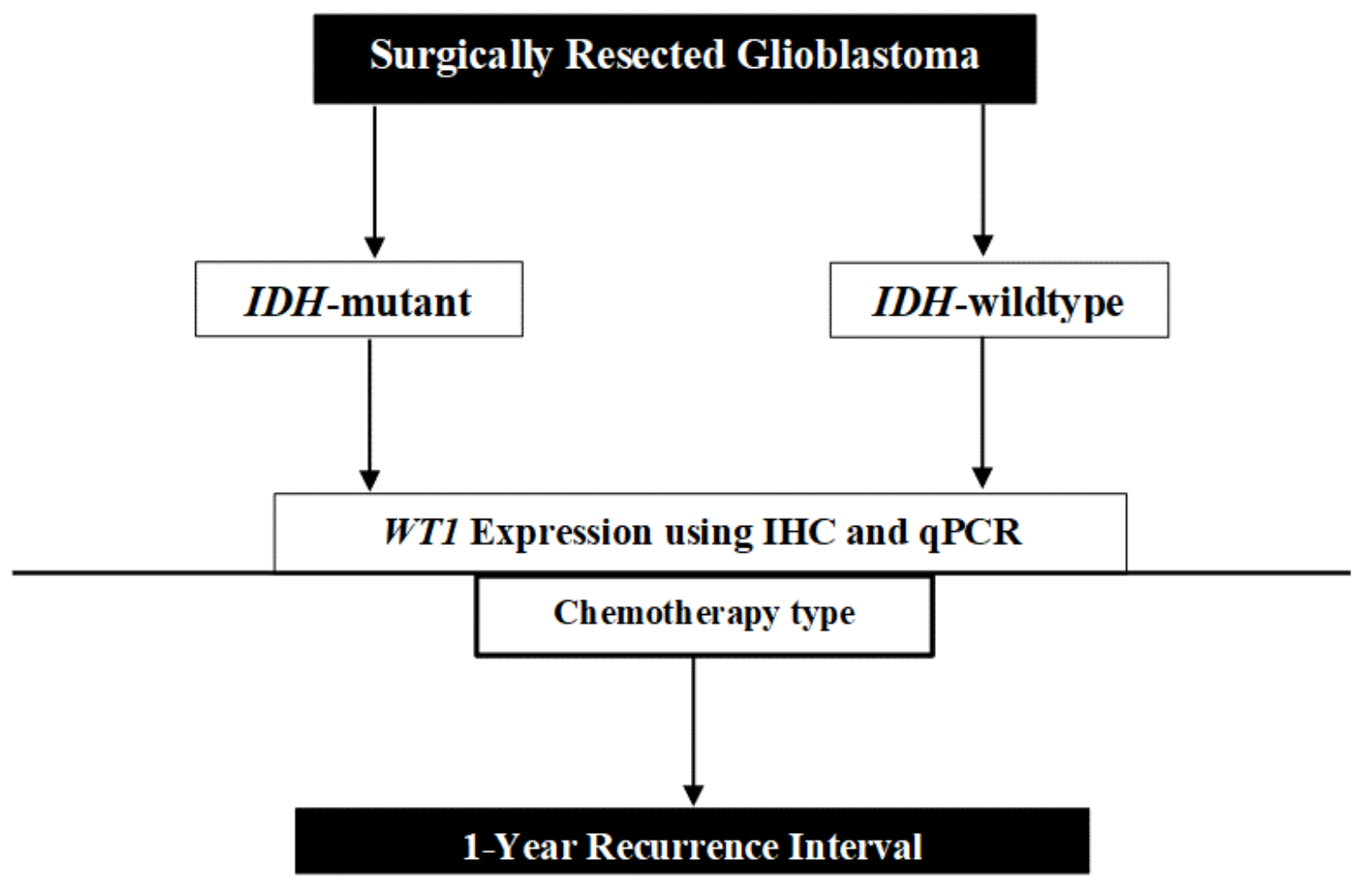

Figure 1

Schematic of the approach used in this study. The samples have been categorized based on ISDH1 mutation and their WT1 expression. Recurrence interval used as determinant factor for patient's outcome.
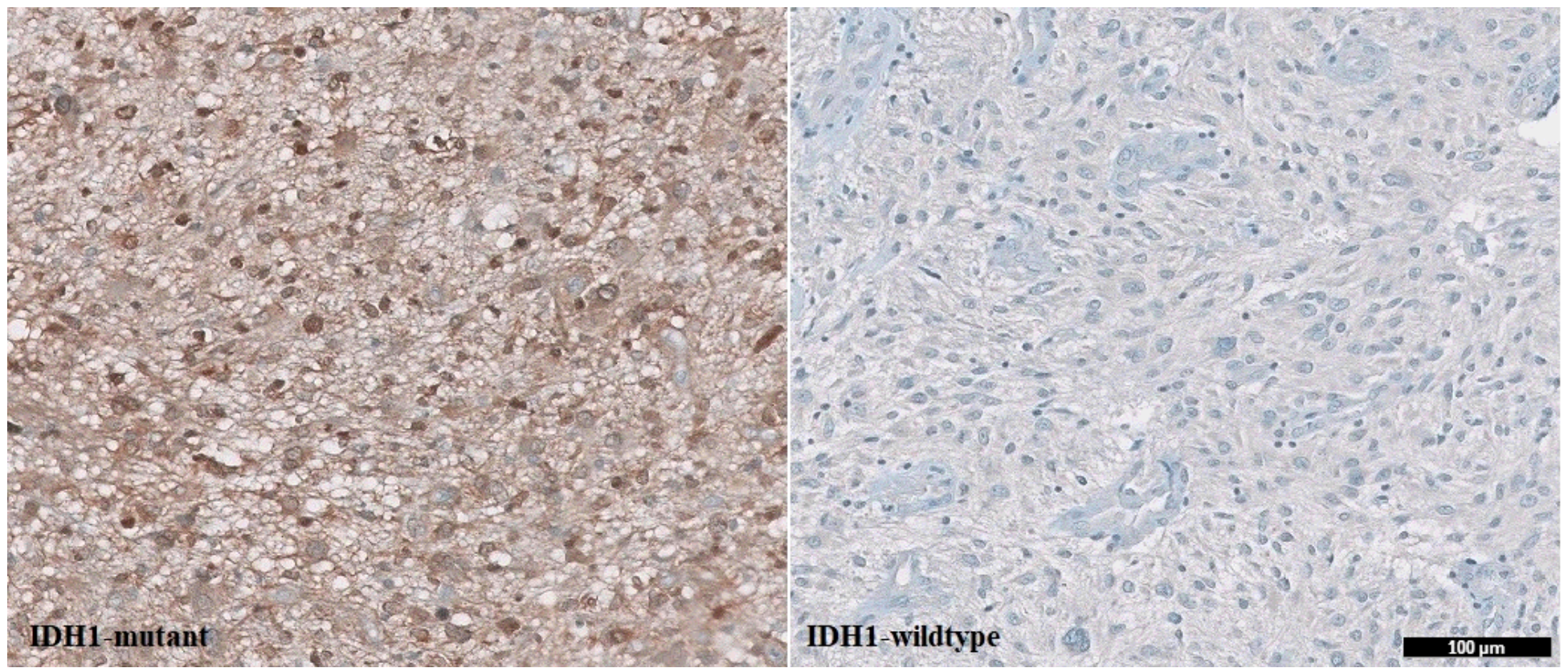

Figure 2 
IDH1 mutation status in glioblastoma using immunohistochemistry (IHC). IDH1 mutation showed positive expression while IDH1-wildtype showed negative expression). Scale bar, $100 \mu \mathrm{m}$.
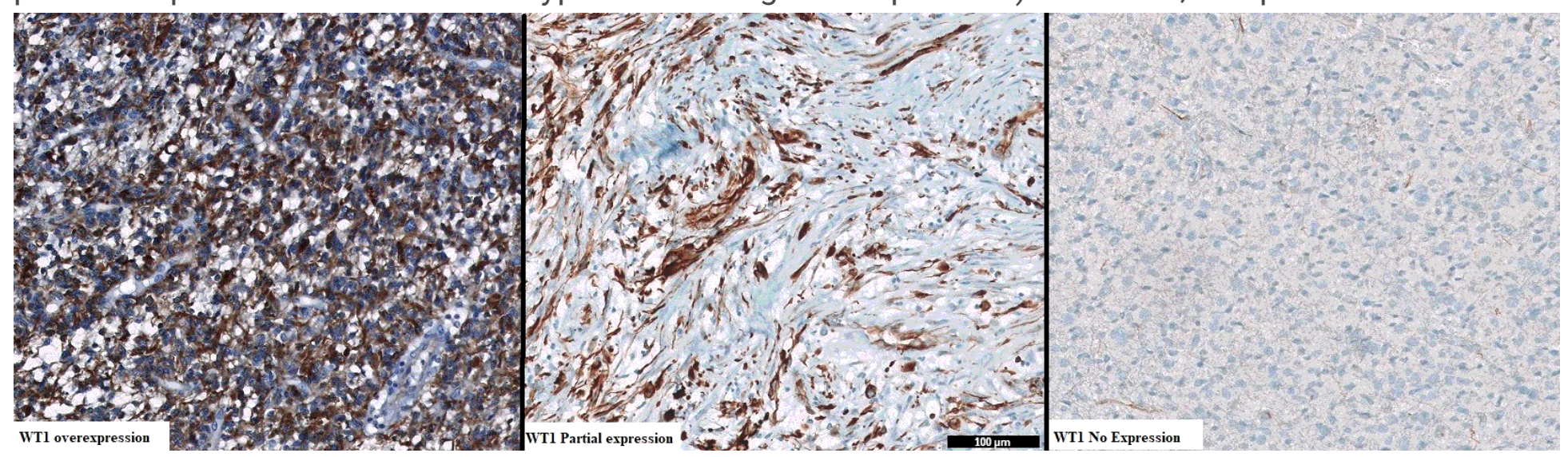

Figure 3

WT1 Expression using the IHC technique. Scale bar, $100 \mu \mathrm{m}$. WT1 protein expression variation (Overexpression, partial expression, and no expression).
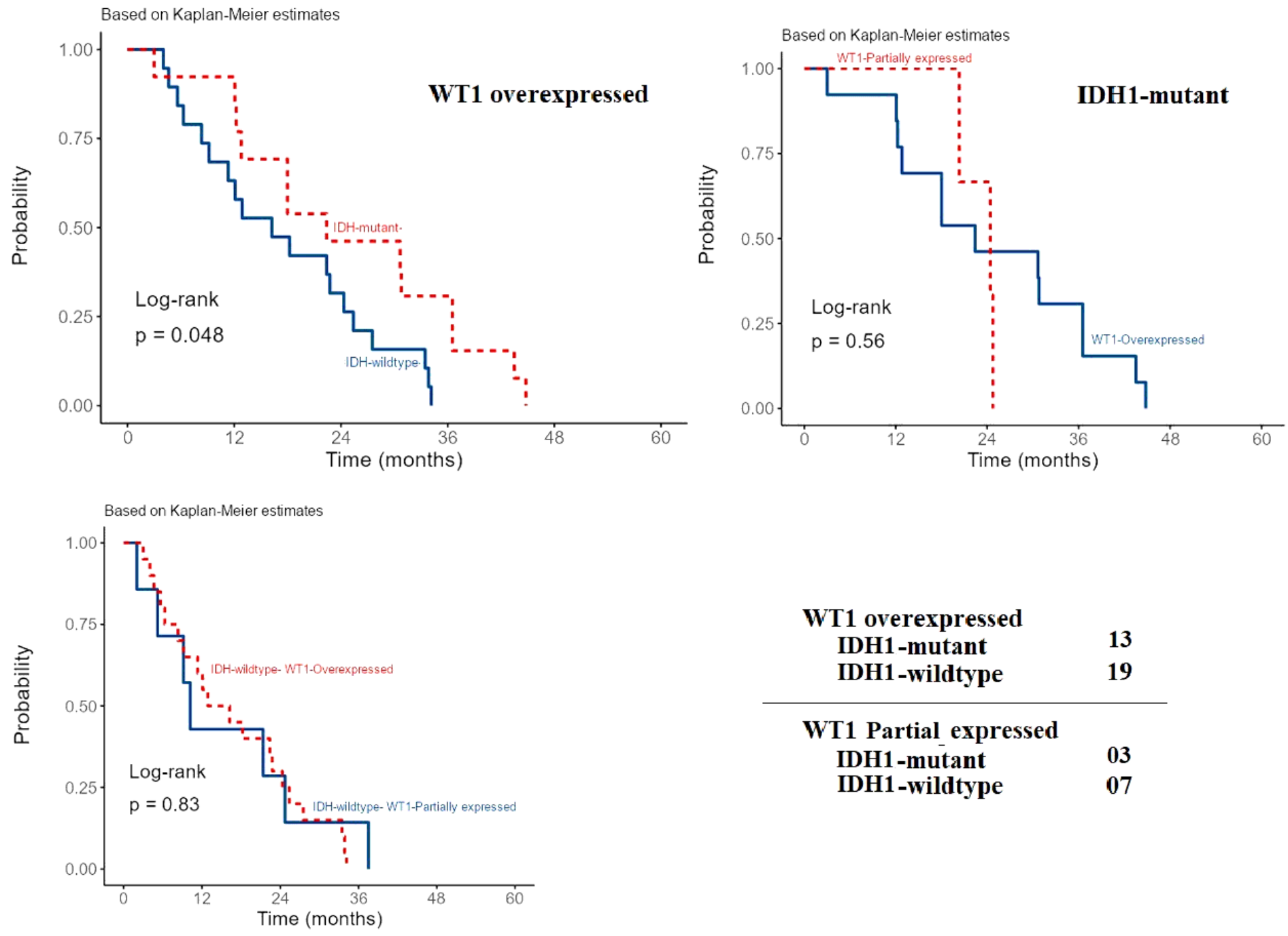


\section{Figure 4}

Recurrence interval among patients with glioblastoma and different IDH1 status and WT1 expression. The recurrence interval among patients with WT1 overexpression significantly differs between wildtype and mutant IDH1 (P-value < 0.048). This significance was not observed among cases with mutant IDH1 and partially expressed or overexpressed WT1 (P-value $=0.56)$ as well as among cases with wildtype IDH1 and WT1 partial or overexpression (P-value $=0.83$. 